\title{
SLOWLY OSCILLATING PERIODIC SOLUTIONS OF AUTONOMOUS STATE-DEPENDENT DELAY EQUATIONS
}

\author{
By \\ Y. Kuang \\ and \\ H.L. Smith
}

IMA Preprint Series \# 754

December 1990 
Abstract. Slowly oscillating periodic solutions are shown to exist for the state-dependent delay equation $x^{\prime}(t)=-f(x(t-\tau(x(t))))$, where $x f(x)>0, x \neq 0, f^{\prime}(0) \tau(0)>\pi / 2$, $f: \mathbf{R} \rightarrow \mathbf{R}, \tau: \mathbf{R} \rightarrow(0, \infty)$ are locally lipschitzian and a certain a priori bound exists for solutions initiating from a restricted set of initial data. Solutions are shown to oscillate if $f^{\prime}(0) \tau(0)>e^{-1}$.

Key words: slowly oscillating periodic solution, state-dependent delay equation, oscillation. 


\section{Introduction}

It has been more than a dozen years since the peak of activity on the question of the existence of periodic, slowly oscillating, solutions of autonomous delay differential equations. Following early work of Jones [J], Wright [Wr] and Grafton [G], the work of Nussbaum [N1, N2] is to be especially noted for providing several new fixed point results and a global bifurcation theorem which are particularly useful for proving the existence of periodic solutions. Other important works include those of Hadeler and Tomiuk [HT], Kaplan and Yorke [KY], Chow [C], Chow and Hale [CH], Alt [A1, A2] and Walther [W1, W2]. See Hale's book [H] for an overview.

To the best of our knowledge, only Nussbaum [N2] and Alt [A2] considered the question of the existence of periodic solutions of autonomous state-dependent delay differential equations. Nussbaum considered a special equation, (0.3) below, in [N2] but did not prove a general result. Alt [A2] considered more complicated, integral threshold-type, state dependent delays which have arisen in various models in epidemiology and structured population models. Alt obtained a theorem for a general class of state-dependent equations on which we comment below.

The lack of results on periodic solutions for state-dependent equations is probably explained by the fact that it is not clear what kinds of state-dependent delays are interesting or natural and on a lack of compelling examples of such equations arising from plausible mathematical models. However, some recent papers of Bélair and Mackey [BM] and Bélair $[B]$ contain some interesting state-dependent delay equations arising in economics and population biology. State dependent delays of threshold type arise naturally in structured population models (see [A1, A2]).

The motivation for this paper stems from consideration of the innocent-looking equation

$$
x^{\prime}(t)=-k x(t-\tau(x(t)))
$$

where $\tau(x)$ is bell-shaped, e.g., $\tau(x)=\alpha e^{-x^{2}}+1-\alpha, 0 \leq \alpha \leq 1$. If $k>\pi / 2$ and $\tau(0)=1$, 
then the zero solution of $(0.1)$ is unstable. A formal linearization would yield

$$
z^{\prime}(t)=-k z(t-1)
$$

which is known to be unstable for $k>\pi / 2$. On the other hand, in case

$$
\lim _{x \rightarrow-\infty} \tau(x)=\lim _{x \rightarrow+\infty} \tau(x)=\tau_{0}>0
$$

then, near infinity, we have the formal linearization

$$
z^{\prime}(t)=-k z\left(t-\tau_{0}\right)
$$

which will be stable if $\tau_{0}<\pi / 2$. If $k>\pi / 2$ and $k \tau_{0}<\pi / 2$, then one would naturally expect the possibility of periodic solutions or other interesting behavior. Figure 1 shows some numerically integrated solutions of (0.1).

As often happens, one thing leads to another, and we are led to a more general equation. Consider the differential delay equation

$$
x^{\prime}(t)=-f(x(t-\tau(x(t))))
$$

where we assume

(F) $f: \mathbf{R} \rightarrow \mathbf{R}$ is locally lipschitzian and

$$
x f(x)>0, \quad x \neq 0 .
$$

(D) $\tau: \mathbf{R} \rightarrow(0, \infty)$ is locally lipschitzian.

In addition, we assume that an a priori bound exists for solutions of (0.2) satisfying certain special initial conditions. More precisely, for $N>0$ let $r=r(N)=\max \{\tau(x): 0 \leq x \leq N\}$. Define

$$
\begin{gathered}
K_{N}=\{\phi:(-\infty, 0] \rightarrow \mathbf{R}: \phi \text { is lipschitzian on }(-\infty, 0], \phi \geq 0, \\
\phi \text { is nondecreasing, } \phi(0) \leq N \text { and } \phi(-r)=0\} .
\end{gathered}
$$


Then our a priori bound assumption is

(B) There exists $M>0, N>0$ such that

$$
-M \leq x(t, \phi) \leq N
$$

for all $t$ in the domain of existence of $x(\cdot, \phi)$, the solution of $(0.2)$ satisfying $x(t, \phi)=\phi(t)$, $t \leq 0$, for every $\phi \in K_{N}$.

Our main result follows.

Theorem A: Let (F), (D) and (B) hold and

$$
\tau(0) \cdot f^{\prime}(0)>\frac{\pi}{2}
$$

Then (0.2) has a nonconstant periodic solution $x(t)$ of period, $\omega$, greater than $2 \tau(0)$, satisfying

$$
-M \leq x(t) \leq N
$$

Furthermore, $x(t)$ has precisely two zeros, $z_{1}, z_{2}$, in $[0, \omega]$, both simple, $0<z_{1}<z_{2}<\omega$, with $z_{2}-z_{1}>\tau(0)$.

We borrow the terminology "slowly oscillating" for the periodic solution guaranteed by the theorem since the separation between consecutive zeros is larger than $\tau(0)$. See Corollary 1.6 for more information concerning the periodic solution.

If $x(t)$ satisfies $(0.2)$, then $y(t)=x(\tau(0) t)$ satisfies $y^{\prime}(t)=-\tau(0) f(y(t-\tau(y(t)) / \tau(0)))$ so we may assume without loss of generality that $\tau(0)=1$. Hereafter, this will be assumed.

Alt observes in a concluding remark in [A2] that his methods can be used to prove the existence of periodic solutions for $(0.2)$ where $\tau(x)=\sigma(|x|), \sigma(0)>0$ and $\sigma$ is nondecreasing.

J. Mallet-Paret and R. Nussbaum have announced very interesting work related to ours at the International Conference on Differential Equations and Applications to Biology and Population Dynamics (Claremont, California, January 1990). They consider a singularly 
perturbed equation of the form

$$
\epsilon x^{\prime}(t)=-f(x(t), x(t-r))
$$

where $r=r(x(t)) \geq 0$ and $f$ and $r$ are given functions. They are able to describe the asymptotic shape of slowly oscillating solutions as $\epsilon$ tends to zero under appropriate conditions on $f$ and $r$. In a private communication, J. Mallet-Paret indicates that they obtain, as part of their work, an existence result for periodic solutions of this equation [MN].

The key observation which we exploit in this paper is that if a solution $x(t)$ of $(0.2)$ satisfies $x^{\prime}\left(t_{0}\right)=0$, then for $t>t_{0}$, the solution "forgets" its history prior to $t_{0}-\tau\left(x\left(t_{0}\right)\right)$, in the sense that $t-\tau(x(t))>t_{0}-\tau\left(x\left(t_{0}\right)\right)$ for all $t>t_{0}$. With the aid of this simple observation, most of the proof of our theorem follows more or less standard lines. We set up a Poincaré-like map from a compact, convex subset of the space of continuous functions on a compact interval into itself, which contains the zero function, and we use a theorem of Browder [Br] on the existence of a nonejective fixed point (we show that 0 is an ejective fixed point). The Theorem is proved in section one.

In section two, we give fairly general sufficient conditions for (B) to hold for some $M, N>$ 0 . These conditions hold, for example, if for some $N>0$,

$$
\left.\tau( \pm N) \operatorname{lip}\right|_{[-N, N]}<1
$$

in which case we may take $M=N$. The notation $\left.\operatorname{lip}\right|_{[-N, N]}$ is used for $\operatorname{ess} \sup \left\{\left|f^{\prime}(x)\right|\right.$ : $-N \leq x \leq N\}$, the smallest lipschitz constant for $\left.f\right|_{[-N, N]}$. In addition, (B) holds for some $M, N>0$ if either $f$ is bounded from below, or from above, and $\tau$ is bounded from above.

All our assumptions hold for (0.1) provided $k>\pi / 2$ and

$$
k \tau( \pm N)<1
$$

for some $N>0$. In particular, if $k \tau_{0}<1$, where $\tau_{0}=\lim _{x \rightarrow+\infty} \tau(x)=\lim _{x \rightarrow-\infty} \tau(x)$, then $(0.1)$ has a nonconstant slowly oscillating periodic solution. If $\tau(x)=\alpha e^{-x^{2}}+(1-\alpha)$, 
$0<\alpha<1$, then Theorem A and Proposition 2.2 imply the existence of a nonconstant periodic solution if $\pi / 2<k<3 /(2(1-\alpha))$.

As another example, consider,

$$
x^{\prime}(t)=-\alpha x(t-1-|x(t)|)\left(1-x^{2}(t)\right)
$$

mentioned by Halanay and Yorke [HY]. For a solution $x(t)$ of $(0.3)$ satisfying $|x(t)|<1$ the change of variables $x(t)=\tanh u(t)$ results in the equation

$$
u^{\prime}(t)=-\alpha \tanh (u(t-\tau(u(t))))
$$

where

$$
\tau(u)=1+|\tanh u|
$$

It is apparent that all the hypotheses of our theorem hold if $\alpha>\pi / 2$ and hence (0.3) has a nontrivial slowly oscillating periodic solution satisfying $-1<x(t)<1$. See Figure 2 for the numerical solution of (0.3).

R. Nussbaum [N2] proved the existence of a periodic solution of the equation (0.3) by working in the space of $C^{1}$ functions on a suitable interval and using a rather nonstandard fixed point theorem. Our result includes (0.3) and our proof appears to be simpler than the one in [N2].

Our result may be applied to the delay logistic equation

$$
x^{\prime}(t)=r x(t)(1-x(t-\sigma(x(t))))
$$

with suitable hypotheses on $\sigma(x)$, following the change of variables $u=\ln x$, which yields

$$
u^{\prime}(t)=-r\left(e^{u(t-r(u(t)))}-1\right)
$$

where $\tau(u)=\sigma\left(e^{u}\right)$.

We expect that our main result generalizes to equations of the form

$$
x^{\prime}(t)=-f(x(t), x(t-\tau(x(t)))),
$$


with suitable conditions on $f$.

In section two we obtain sufficient conditions for solutions $x(t)$, defined for $t \geq 0$, to oscillate, i.e., to have arbitrarily large zeros. Our result is the following.

Theorem B: If $(F)$ and $(D)$ hold, $\tau(0)=1$ and

$$
f^{\prime}(0)>e^{-1}
$$

then every solution $x(t)$ of (0.2), defined for $t \geq 0$ and for which $\lim _{t \rightarrow \infty} t-\tau(x(t))=\infty$, must oscillate.

This research has, in some way, been motivated by a renewed interest in state-dependent delay equations by some participants of the recent period of concentration on Mathematical Physiology and Differential Delay Equations, organized chiefly by M. Mackey, at the IMA in the spring of 1990 . The second author would like to thank the organizers of this period of concentration and also the staff at IMA for a very productive and enjoyable visit.

The authors are grateful to E. Lo for providing the numerical solutions displayed in Figures 1 and 2, using the numerical schemes developed in [JL].

\section{Existence of Periodic Solutions.}

In this section we prove the existence of periodic solutions of

$$
x^{\prime}(t)=-f(x(t-\tau(x(t))))
$$

We assume throughout this section that the following hold.

(F) $f: \mathbf{R} \rightarrow \mathbf{R}$ is locally lipschitzian and satisfies

$$
x f(x)>0, \quad x \neq 0 .
$$

(D) $\tau: \mathrm{R} \rightarrow(0, \infty)$ is locally lipschitzian and $\tau(0)=1$.

Lemma 1.1: For each bounded, lipschitzian function $\phi:(-\infty, 0] \rightarrow \mathbf{R}$ there exists a unique noncontinuable solution $x(t, \phi)$ of $(1.1)$ defined for $t \in[0, \omega), 0<\omega \leq \infty, x(t)=\phi(t)$ for 
$t \leq 0$. If $\omega<\infty$, then there exists $t_{n} \nearrow \omega$ such that $\left|x\left(t_{n}\right)\right| \rightarrow \infty$ as $n \rightarrow \infty$. If $\liminf _{|x| \rightarrow \infty} \tau(x)>0$, then $\omega=+\infty$.

Proof: These are well-known results. See Halanay and Yorke [HY], Grossman and York [GY], Driver [D]. Uniqueness follows from [HY, Prop. 1.3]. Since the right-hand side is bounded on bounded subsets of the space of bounded, lipschitzian functions on $(-\infty, 0]$, the behavior of $x(t)$ as $t \rightarrow \omega$ follows from [HY, Proposition 1.2]. In the case of (1.1) it is especially easy to see. If $|x(t)|$ is bounded on its maximal interval of existence, then its derivative is also bounded and thus $\lim _{t \rightarrow \omega^{-}} x(t)$ exists. The solution can then be extended to a larger interval.

Alternatively, the entire lemma may easily be obtained by a careful application of the method of steps.

Given a bounded, lipschitzian function $\phi$ on $(-\infty, 0]$ we write $x(t, \phi)$ for the noncontinuable solution of (1.1) for $t \geq 0$ satisfying $x(t)=\phi(t)$ for $t \leq 0$. We will use this notation in case $\phi$ is defined and lipschitz continuous on some compact interval $[-r, 0]$ provided it is known that $t-\tau(x(t, \phi)) \geq-r$ for $t \geq 0$.

Lemma 1.2: Let $x(t, \phi)$ be a solution of (1.1) and suppose $x^{\prime}\left(t_{0}\right)=0$ for some $t_{0} \geq 0$. Then $t-\tau(x(t))>t_{0}-\tau\left(x\left(t_{0}\right)\right)$ for all $t>t_{0}$. If $t_{0}>0$, then $t-\tau(x(t))<t_{0}-\tau\left(x\left(t_{0}\right)\right)$ for $0 \leq t<t_{0}$.

Proof: By $(\mathrm{F}), x\left(t_{0}-\tau\left(x\left(t_{0}\right)\right)\right)=0$. Since $\tau$ is locally lipschitzian and $x^{\prime}\left(t_{0}\right)=0$, there exists $\eta>0$ such that for $0<t-t_{0}<\eta$,

$$
\left|\tau(x(t))-\tau\left(x\left(t_{0}\right)\right)\right|<t-t_{0}
$$

Thus, for $t_{0}<t<t_{0}+\eta$,

$$
t-t_{0}>\tau(x(t))-\tau\left(x\left(t_{0}\right)\right),
$$

or

$$
t-\tau(x(t))>t_{0}-\tau\left(x\left(t_{0}\right)\right) .
$$


If the assertion of the Lemma is false, there is a smallest $t_{1}>t_{0}$ such that

$$
t_{1}-\tau\left(x\left(t_{1}\right)\right)=t_{0}-\tau\left(x\left(t_{0}\right)\right)<t-\tau(x(t))
$$

for $t_{0}<t<t_{1}$. Obviously, $x^{\prime}\left(t_{1}\right)=0$ since $f\left(x\left(t_{1}-\tau\left(x\left(t_{1}\right)\right)\right)=f\left(x\left(t_{0}-\tau\left(x\left(t_{0}\right)\right)\right)=\right.\right.$ $x^{\prime}\left(t_{0}\right)=0$ and arguing as above, there exists $\delta>0$ such that for $0<\left|t-t_{1}\right|<\delta$,

$$
\left|\tau(x(t))-\tau\left(x\left(t_{1}\right)\right)\right|<\left|t-t_{1}\right|
$$

But this implies that for $t_{1}-\delta<t<t_{1}$,

$$
t_{1}-t>\tau\left(x\left(t_{1}\right)\right)-\tau(x(t))
$$

which contradicts (1.2). This completes the proof.

Lemma 1.2 has an important consequence which we exploit later on. Namely, if $x^{\prime}\left(t_{0}\right)=0$ for some $t_{0} \geq 0$, then the solution $x(t)$ for $t \geq t_{0}$ depends only on $x(s)$ for $s$ in the interval $\left[t_{0}-\tau\left(x\left(t_{0}\right)\right), t_{0}\right]$. The solution "forgets" some of its past history.

For $N>0$, let $r=r(N)=\max \{\tau(x): 0 \leq x \leq N\}$ and define

$\tilde{K}_{N}=\left\{\phi \in C_{r}: \phi(-r)=0, \phi \geq 0, \phi\right.$ is nondecreasing, lip $\phi<\infty$ and $\left.\phi(0) \leq N\right\}$.

$C_{r} \equiv C([-r, 0], \mathrm{R})$ denotes the space of continuous functions on $[-r, 0]$ with the uniform norm $\|\cdot\|$. We introduce the following hypothesis.

(B) There exists $N>0, M>0$ such that

$$
-M \leq x(t ; \phi) \leq N
$$

for all $t$ in the domain of $x(\cdot, \phi)$, and for all $\phi \in \tilde{K}_{N}$.

If (B) holds for some $N>0, M>0$, then necessarily the domain of $x(\cdot, \phi)=[0, \infty)$ for every $\phi \in \tilde{K}_{N}$, by Lemma 1.1, and hence the inequality holds for all $t \geq 0$. We show below that $t-\tau(x(t)) \geq-r$ for all $t \geq 0$ for $\phi \in \tilde{K}_{N}$. Set $R=\max \{\tau(x):-M \leq x \leq N\}$ so $R \geq r \geq 1$, and $E=\max \{f(x): 0 \leq x \leq N\}$. 
Proposition 1.3. Assume that (B) holds, $f^{\prime}(0)>1, \phi \in \tilde{K}_{N} \backslash 0$, and $x(t)=x(t, \phi)$. Then

(i) There exists $z_{1}=z_{1}(\phi) \geq(\phi(0)) / E$ such that $x(t)>0$ and $x^{\prime}(t) \leq 0$ on $\left[0, z_{1}\right)$, $x\left(z_{1}\right)=0$ and $x^{\prime}\left(z_{1}\right)<0$.

(ii) There exists $P=P(N)>0$ such that $z_{1}(\phi) \leq P$ for all $\phi \in \tilde{K}_{N} \backslash 0$.

(iii) There exists $t_{1}=t_{1}(\phi) \in\left(z_{1}, z_{1}+R\right]$ such that $x^{\prime}(t)<0$ on $\left[z_{1}, t_{1}\right)$ and $x^{\prime}\left(t_{1}\right)=0$.

(iv) There exists $z_{2}=z_{2}(\phi)>t_{1}$ such that $x(t)<0, x^{\prime}(t)>0$ on $\left(t_{1}, z_{2}\right), x\left(z_{2}\right)=0$ and $x^{\prime}\left(z_{2}\right)>0$. Moreover, $z_{2}-z_{1}>1$.

(v) There exists $Q=Q(M)>0$ such that $z_{2}(\phi) \leq Q$ for all $\phi \in \tilde{K}_{N} \backslash 0$.

(vi) There exists $t_{2}=t_{2}(\phi) \in\left(z_{2}, z_{2}+r\right]$ such that $x(t)>0, x^{\prime}(t)>0$ on $\left(z_{2}, t_{2}\right)$ and $x^{\prime}\left(t_{2}\right)=0$.

Proof: Clearly $x^{\prime}(t) \leq 0$ for all $t \in\left[0, z_{1}\right]$ where we take $z_{1}=+\infty$ if $x$ never vanishes. Define $F(x)=\min \{f(u): x \leq u \leq N\}$ for $0 \leq x \leq N$ so that $F$ is lipschitz, $F(x)>0$ for $x>0, F$ is increasing and $F(0)=0$. If $z_{1}>r$, then for $r \leq t \leq z_{1}$,

$$
\begin{aligned}
x^{\prime}(t) & =-f(x(t-\tau(x(t)))) \leq-F(x(t-\tau(x(t)))) \\
& \leq-F(x(t))
\end{aligned}
$$

since $t-\tau(x(t))>0$ and $x(t-\tau(x(t))) \geq x(t)$. From standard differential inequality arguments,

$$
x(t) \leq u(t), \quad r \leq t \leq z_{1}
$$

where

$$
u^{\prime}(t)=-F(u(t)), \quad u(r)=N
$$

Observe that $u(t)$ is independent of $\phi \in \tilde{K}_{N}$ and that $\lim _{t \rightarrow \infty} u(t)=0$.

Let $k$ satisfy $f^{\prime}(0)>k>1$ and choose $\delta>0$ such that $f(x)>k x$ for $0<x \leq \delta$. Choose $\delta$ smaller if necessary so that $k \tau(x) \geq 1$ for $0 \leq x \leq \delta$. From the previous paragraph, there exists $T>r$, depending only on $N$, such that $x(t) \leq \delta$ for $T \leq t \leq z_{1}$, if $T<z_{1}$. If 
$r+T+k^{-1}<z_{1}$, then for $r+T \leq t \leq r+T+k^{-1}$

$$
x^{\prime}(t)<-k x(t-\tau(x(t))) \leq-k x(r+T)
$$

since, for the above range of $t$,

$$
T<t-\tau(x(t)) \leq t-k^{-1} \leq r+T
$$

and $x$ is decreasing. Hence

$$
x\left(r+T+k^{-1}\right)<x(r+T)-k x(r+T) k^{-1}=0,
$$

a contradiction to our assumption that $r+T+k^{-1}<z_{1}$. We conclude that $z_{1} \leq r+T+k^{-1}$. Since $x^{\prime}(t) \geq-E$ for $0 \leq t \leq z_{1}$, it follows immediately that $z_{1} \geq \phi(0) / E$. This establishes (i) and (ii) except for the assertion that $x^{\prime}\left(z_{1}\right)<0$.

Assume that $\phi(t)=0$ for $-r \leq t \leq d, d<0$, and $\phi(t)>0$ on $(d, 0]$. If $-\tau(\phi(0))>d$, then $x^{\prime}(0)<0$ and we claim that $t-\tau(x(t))>d$ for all $t>0$. The argument is exactly like that in Lemma 1.2. If $t_{0}>0$ is the smallest positive time at which $t-\tau(x(t))=d$, then $x^{\prime}\left(t_{0}\right)=0$ and so there exists $\eta>0$ such that

$$
\left|\tau(x(t))-\tau\left(x\left(t_{0}\right)\right)\right|<\left|t-t_{0}\right|
$$

if $\left|t-t_{0}\right|<\eta$. But then, for $t_{0}-\eta<t<t_{0}$,

$$
t_{0}-t>\tau\left(x\left(t_{0}\right)\right)-\tau(x(t))
$$

and hence

$$
t-\tau(x(t))<t_{0}-\tau\left(x\left(t_{0}\right)\right)=d
$$

Since $0-\tau(\phi(0))>d$, the above inequality and the Intermediate Value Theorem imply the existence of $t^{*} \in\left(0, t_{0}\right)$ at which $t^{*}-\tau\left(x\left(t^{*}\right)\right)=d$, contradicting the minimality of $t_{0}$. Hence, our claim is valid and it follows that $x^{\prime}(t)<0$ for $0 \leq t \leq z_{1}$. If $-\tau(\phi(0))=d$, then $x^{\prime}(0)=0$ and, by Lemma $1.2, t-\tau(x(t))>d$ for all $t>0$. Hence, again, $x^{\prime}(t)<0$ for 
$0<t \leq z_{1}$. If $-\tau(\phi(0))<d$, then $x^{\prime}(t)=0$ so long as $t-\tau(x(t)) \leq d$. Lemma 1.2 implies that the set of such $t$ is an interval $\left[0, t_{0}\right], t_{0}>0$, on which $x(t) \equiv \phi(0)$. It follows that $t_{0}-\tau(\phi(0))=d$ or $t_{0}=d+\tau(\phi(0))$. For $t>t_{0}, t-\tau(x(t))>d$, by Lemma 1.2, and thus $x^{\prime}(t)<0$ for $\left(t_{0}, z_{1}\right]$. This completes the proof of (i) and (ii).

By Lemma 1.2 and the arguments above, $x^{\prime}(t)<0$ for $t \geq z_{1}$ until time $t_{1}$ at which $t_{1}-\tau\left(x\left(t_{1}\right)\right)=z_{1}$. By (B), $t_{1}=z_{1}+\tau\left(x\left(t_{1}\right)\right) \leq R+z_{1}$ and $x^{\prime}\left(t_{1}\right)=0$. By Lemma 1.2, $t-\tau(x(t))>z_{1}$ for $t>t_{1}$ implying that $x^{\prime}(t)>0$ for $t_{1}<t \leq z_{2}$, where $z_{2}$ is the second positive zero of $x(t)$ or $+\infty$ if no such zero exists.

But $x(t)$ must have a second positive zero by arguments identical to those yielding the existence of its first zero. Indeed, by the remark following Lemma 1.2, $x(t)$, for $t \geq t_{1}$ is determined by the values $x(s)$ for $s$ in the interval $\left[z_{1}, t_{1}\right]$, which are nonpositive and bounded below by $-M$. Thus, one can argue exactly as for the existence of $z_{1}$ and its upper bound $P$, to show the existence of the second zero $z_{2}$ satisfying $z_{2}(\phi) \leq Q$ for all $\phi \in \tilde{K}_{N} \backslash 0$. Thus (iv) and (v) hold. Since $x^{\prime}\left(z_{2}\right)>0, x\left(z_{2}-\tau\left(x\left(z_{2}\right)\right)\right)=x\left(z_{2}-1\right)<0$ so $z_{2}-1>z_{1}$.

As $x^{\prime}\left(z_{2}\right)>0$ and $t-\tau(x(t))>z_{1}$ for $t>t_{1}$ it follows that $x^{\prime}(t)>0$ for $t \geq z_{2}$ until such time $t_{2}$ at which $t_{2}-\tau\left(x\left(t_{2}\right)\right)=z_{2}$. By (B), such a $t_{2}$ exists and $t_{2}=z_{2}+\tau\left(x\left(t_{2}\right)\right) \leq z_{2}+r$. This establishes (vi).

It follows from Proposition 1.3 that $x(t, \phi)$ has infinitely many zeros $z_{n}(\phi), n \geq 1$, which are simple and $z_{n}-z_{n-1}>1$ for $n \geq 2$, if (B) holds, $\phi \in \tilde{K}_{N} \backslash 0$ and $f^{\prime}(0)>1$. Moreover, $x^{\prime}(t, \phi)=0$ for $t \geq z_{1}$ only at points $t_{n}(\phi), n \geq 1$, satisfying $z_{n}<t_{n}<z_{n+1}, n \geq 1$, where $t_{n}-\tau\left(x\left(t_{n}\right)\right)=z_{n}$ and $x^{\prime}(t)>0$ on $\left(t_{n}, t_{n+1}\right)$, for $n \geq 1, n$ odd, and $x^{\prime}(t)<0$ on $\left[z_{1}, t_{1}\right)$ and on $\left(t_{n}, t_{n+1}\right), n \geq 2, n$ even. Figure 3 summarizes this information.

We note for later use that the hypothesis (B) was not used in Proposition 1.3 (i) and (ii). The lower bound $-M$ on the solution was used to show that $t_{1}<+\infty$ and to obtain the existence of $z_{2}$. The upper bound $N$ was then required to show that $t_{2}<\infty$. 
Assuming that (B) holds and that $L=\max \{|f(x)|:-M \leq x \leq N\}$, we define

$$
K=\left\{\phi \in \tilde{K}_{N}: \operatorname{lip} \phi \leq L\right\}
$$

Observe that $K$ is a compact, convex subset of $C_{r}$ containing the zero function and that $\operatorname{lip} x(\cdot, \phi) \leq L$ for all $\phi \in \tilde{K}_{N}$. Define the map $T: K \rightarrow C_{r}$ by:

$$
(T \phi)(\theta)=\left[x\left(t_{2}(\phi)+\theta, \phi\right)\right]^{+},-r \leq \theta \leq 0,
$$

if $\phi \in K \backslash 0$ and $T 0=0$. For $\psi \in \mathbf{R}, \psi^{+}=\max \{0, \psi\}$.

The following will be used to establish the continuity of $T$.

Lemma 1.4: Assume that (B) holds. Then there exists $A>0$ such that

$$
|x(t, \phi)-x(t, \psi)| \leq\|\phi-\psi\| e^{A t}, \quad t \geq 0
$$

for $\phi, \psi \in K$.

Proof: This is a simple Gronwall estimate. Let $p=\left.\operatorname{lip} f\right|_{[-M, N]}, q=\left.\operatorname{lip} \tau\right|_{[-M, N]}$ and recall that $\operatorname{lip} x(\cdot, \phi) \leq L$ for any $\phi \in K$. Put $x(t)=x(t, \phi)$ and $y(t)=x(t, \psi)$, for $\phi, \psi \in K$. Then

$$
\begin{aligned}
|x(t)-y(t)| \leq & |x(0)-y(0)|+\int_{0}^{t}|f(x(s-\tau(x(s))))-f(y(s-\tau(y(s))))| d s \\
\leq & \|\phi-\psi\|+\int_{0}^{t} p|x(s-\tau(x(s)))-y(s-\tau(y(s)))| d s \\
\leq & \quad+\mid x\left(s-\psi-\psi \|+\int_{0}^{t} p[L q|x(s)-y(s)|\right. \\
& \left.\left.\left.\leq\|\phi-\psi\|+\int_{0}^{t} p[L q+1] Z(s)\right)\right)-y(s-\tau(y(s))) \|\right] d s
\end{aligned}
$$

where $Z(t)=\sup _{s \leq t}|x(s)-y(s)|$. Hence,

$$
Z(t) \leq\|\phi-\psi\|+\int_{0}^{t} A Z(s) d s
$$

where $A=p[L q+1]$. The assertion follows from Gronwall's inequality. 
Proposition 1.5: $T$ maps $K$ continuously into $K$ with respect to the $C_{r}$ topology. If $T \phi=\phi \in K \backslash 0$, then $x(t, \phi)$ is a periodic solution of period $t_{2}(\phi)$.

Proof: By Proposition 1.3, (iv) and (vi), $x\left(t_{2}(\phi)+\theta, \phi\right)>0$ and $x^{\prime}\left(t_{2}(\phi)+\theta, \phi\right)>0$ for $-r \leq z_{2}-t_{2}<\theta<0$ and $\left[x\left(t_{2}(\phi)+\theta, \phi\right)\right]^{+}=0$ for $-r \leq \theta \leq z_{2}-t_{2}$. Hence, $T \phi(-r)=0$, $T \phi \geq 0$ and $T \phi$ is nondecreasing on $[-r, 0]$. (B) implies $(T \phi)(0)=x\left(t_{2}(\phi), \phi\right) \leq N$. Except possibly for a simple point, $T \phi$ is differentiable and $\left|(T \phi)^{\prime}\right| \leq L$. Thus, $T \phi \in K$.

Suppose $T \phi=\phi \in K \backslash 0$. By the remark following Lemma 1.2, $x(t, \phi)$, for $t \geq t_{2}(\phi)$, depends only on the values of $x(s)$ for $s$ in the interval $\left[z_{2}, t_{2}\right]$, that is, $x(t), t \geq t_{2}$, depends only on $x\left(t_{2}+\theta\right)$ for $z_{2}-t_{2} \leq \theta \leq 0$. Since $T \phi=\phi, \phi(\theta)=x\left(t_{2}+\theta\right)$ for $z_{2}-t_{2} \leq \theta \leq 0$, and so by uniqueness of solutions and the autonomous nature of $(1.1), x(t, \phi)$ is periodic of period $t_{2}(\phi)$.

Lemma 1.4 together with Proposition 1.3 (v) immediately imply the continuity of $T$ at $\phi=0$. Let $\phi \in K \backslash 0$ and $z_{i}=z_{i}(\phi), i=1,2$. Given $\epsilon, 0<\epsilon<\left(z_{2}-z_{1}\right) / 2$, choose $\delta=\inf \left\{|x(t, \phi)|: t \in\left[0, z_{1}-\epsilon\right] \cup\left[z_{1}+\epsilon, z_{2}-\epsilon\right]\right\}$. By Lemma 1.4, there exists $\eta>0$ such that if $\psi \in K$ and $\|\psi-\phi\|<\eta$, then $|x(t, \phi)-x(t, \psi)|<\delta / 2$ on $\left[0, z_{2}\right]$. Hence, $x(t, \psi)>\delta / 2$ on $\left[0, z_{1}-\epsilon\right]$ and $x(t, \psi)<-\delta / 2$ on $\left[z_{1}+\epsilon, z_{2}-\epsilon\right]$ so $x(t, \psi)$ has its first zero $z_{1}(\psi) \in\left[z_{1}-\epsilon, z_{1}+\epsilon\right]$. This establishes the continuity of $z_{1}: K \rightarrow(0, \infty)$ at $\phi$. Similar arguments show that $z_{2}$ is continuous on $K \backslash 0$. Recall that $t_{2}(\phi)-\tau\left(x\left(t_{2}(\phi), \phi\right)\right)=z_{2}(\phi)$ for $\phi \in K \backslash 0$ and that $t_{2}(\phi)$ is the unique time $t$ at which $t-\tau(x(t, \phi))=z_{2}$. This fact, together with the estimate $t_{2}(\phi) \leq Q+r$ from Proposition 1.3 (v) and (vi) immediately imply the continuity of $t_{2}: K \backslash 0 \rightarrow(0, Q+r]$. Thus the map $\phi \mapsto x_{t_{2}(\phi)}(\phi)$ from $K \backslash 0$ into $C_{r}$ is continuous. The continuity of $T$ follows from the continuity of $\phi \mapsto \phi^{+}$.

We remark that $T$ is not continuous with respect to the usual Lipschitz norm on the space of Lipschitz functions on $[-r, 0]$. This is because the operation of taking the positive part of a function fails to be continuous in this space.

Corollary 1.6: If $T \phi=\phi \in K \backslash\{0\}$, then the $t_{2}(\phi)$-periodic solution $x(t, \phi)$ has the 


\section{following properties:}

(i) $x(t)$ has precisely two zeros in $\left[0, t_{2}(\phi)\right]$, namely $z_{1}$ and $z_{2}, 0<z_{1}<z_{2}<t_{2}(\phi)$, both of which are simple. Moreover, $z_{2}-z_{1}>1$ and $t_{2}>2$.

(ii) $x(t)$ has two extrema in $\left(0, t_{2}(\phi)\right]$, namely $t_{1}$ and $t_{2}, 0<z_{1}<t_{1}<z_{2}<t_{2} . x^{\prime}(t)<0$ on $\left(0, t_{1}\right)$ and $x^{\prime}(t)>0$ on $\left(t_{1}, t_{2}\right)$.

(iii) $-M \leq x(t) \leq N$ for all $t$.

Proof: Most of the assertions are obvious from Proposition 1.3, Proposition 1.5 and the periodicity of $x(t, \phi)$. Note that as $x^{\prime}(t)<0$ for $t \in\left(t_{2}, z_{3}\right]$ it follows that $x^{\prime}(t)<0$ on $\left(0, t_{1}\right)$. Since $x(t)$ is periodic and $z_{2}-z_{1}>1$, we have $t_{2}=z_{3}-z_{1}=z_{3}-z_{2}+z_{2}-z_{1}>2$.

It follows from Corollary 1.6 that consecutive zeros of the periodic solution $x(t, \phi)$ are separated by more than one time unit.

The next two results are patterned after similar ones in Nussbaum [N2, Lemmas 2.6 and 2.8]. According to Nussbaum, the main idea for the next lemma is due to Wright [Wr].

Lemma 1.7: Assume that (B) and $f^{\prime}(0)>\pi / 2$ hold. Then there exists a positive number $a$, independent of $\phi$, such that for any $\phi \in K \backslash 0$,

$$
\sup _{t \geq z_{n}}|x(t, \phi)| \geq a
$$

for all sufficiently large zeros $z_{n}$ of $x(t, \phi)$.

Proof: We express (1.1) as

$$
x^{\prime}(t)=-\alpha x(t-1)+g\left(x_{t}\right)+h\left(x_{t}\right)
$$

where $\alpha=f^{\prime}(0), g\left(x_{t}\right)=\alpha x(t-1)-f(x(t-1))$ and $h\left(x_{t}\right)=f(x(t-1))-f(x(t-\tau(x(t))))$. Let $\lambda=\mu+i v$ be the complex root of $\lambda+\alpha e^{-\lambda}=0$ satisfying $\mu>0$ and $0<v<\pi$ (see, e.g., [H, Lemma 11.4.1]). Choose a positive number $\epsilon$ such that $\epsilon<(1 / 2) \mu \cos (v / 2)$. Choose $a>0$ such that if $|x| \leq a$ then $|\alpha x-f(x)| \leq(\epsilon / 2)|x|$. If $\phi \in K \backslash 0$, then $-M \leq x(t, \phi) \leq N$ 
by (B) so we may estimate $h\left(x_{t}\right)$ along the solution $x(t, \phi)$ as follows.

$$
\begin{aligned}
\left|h\left(x_{t}\right)\right| & \leq p|x(t-1)-x(t-\tau(x(t)))| \\
& \leq p\left|x^{\prime}(s)\right||\tau(x(t))-1| \\
& \leq p q|f(x(s-\tau(x(s))))||x(t)| \\
& \leq p^{2} q|x(s-\tau(x(s)))||x(t)|
\end{aligned}
$$

where $p=\left.\operatorname{lip} f\right|_{[-M, N]}, q=\left.\operatorname{lip} \tau\right|_{[-M, N]}$ and $s=s(t)$ lies between $t-1$ and $t-\tau(x(t))$. We assume $t>R$ so $t-\tau(x(t))>0$. Then $s>t-R$ and $s-\tau(x(s))>t-2 R$.

Choose $a$ smaller, if necessary, so that $a p^{2} q \leq \epsilon / 2$. Then

$$
\left|g\left(x_{t}\right)+h\left(x_{t}\right)\right| \leq \frac{\epsilon}{2}[|x(t)|+|x(t-1)|]
$$

provided $|x(t)| \leq a$ and $\mid x(s-\tau(x(s)) \mid \leq a$ where $s=s(t)$ as above.

Finally, choose $a$ smaller, if necessary, so that $a p q \leq 1 / 3 \cos (v / 2) e^{-\mu / 2} \leq 1 / 3$ and $q a<1 / 2$.

If $\sup _{t \geq z_{n}}|x(t, \phi)| \geq a$ fails to hold for some zero $z$ of $x(t, \phi)$, then $\sup _{t \geq z}|x(t, \phi)|=\delta<$ $a$. Since $x$ attains its extrema on $[z, \infty)$ at the $t_{n} \geq z$, there exists some $t_{n} \geq z$ such that $\left|x\left(t_{n}\right)\right| \geq(3 / 4) \delta$. We assume $x\left(t_{n}\right)>0$, the other case is similar. Now

$$
\begin{aligned}
\left|\frac{x\left(z_{n}+1\right)-x\left(t_{n}\right)}{x\left(t_{n}\right)}\right| & =\frac{\left|x^{\prime}(s)\right|\left|z_{n}+1-t_{n}\right|}{\left|x\left(t_{n}\right)\right|} \leq \frac{p|x(s-\tau(x(s)))|\left|1-\tau\left(x\left(t_{n}\right)\right)\right|}{x\left(t_{n}\right)} \\
& \leq a p \frac{\left|\tau(0)-\tau\left(x\left(t_{n}\right)\right)\right|}{x\left(t_{n}\right)} \\
& \leq a p q \leq \frac{1}{3} .
\end{aligned}
$$

Hence,

$$
\left|x\left(1+z_{n}\right)\right| \geq\left|x\left(t_{n}\right)\right|-\frac{\left|x\left(1+z_{n}\right)-x\left(t_{n}\right)\right|}{\left|x\left(t_{n}\right)\right|} \cdot\left|x\left(t_{n}\right)\right| \geq \frac{3}{4} \delta\left(1-\frac{1}{3}\right)=\frac{\delta}{2} .
$$

Putting $T=1+z_{n}$, the remainder of the argument follows [N2, Lemma 2.6]. We give the argument as there are some changes. Assume $x(T)>0$, the other case is similar. If $\lambda$ is the root defined above, integrate by parts to obtain

$$
\int_{T}^{\infty} x^{\prime}(t) \exp (-\lambda t) d t=-x(T) \exp (-\lambda T)+\lambda \int_{T}^{\infty} x(t) \exp (-\lambda t) d t .
$$


Replacing $x^{\prime}(t)$ in (1.4) by the right side of (1.3), we obtain

$$
\begin{gathered}
\int_{T}^{\infty} x^{\prime}(t) \exp (-\lambda t) d t=-\alpha \int_{T}^{\infty} x(t-1) \exp (-\lambda t) d t+\int_{T}^{\infty}\left[g\left(x_{t}\right)+h\left(x_{t}\right)\right] \exp (-\lambda t) d t \\
\quad=-\alpha \exp (-\lambda) \int_{T}^{\infty} x(t) \exp (-\lambda t) d t-\alpha \int_{T}^{T+1} x(t-1) \exp (-\lambda t) d t+I,
\end{gathered}
$$

where $I$ represents the integral containing $g\left(x_{t}\right)+h\left(x_{t}\right)$. Using $\lambda+\alpha \exp (-\lambda)=0$, setting (1.4) equal to (1.5) and changing variables in the integral from $T$ to $T+1$ yields

$$
-x(T) \exp (-\lambda T)+\alpha \exp (-\lambda) \int_{T-1}^{T} x(t) \exp (-\lambda t) d t=I .
$$

Integrating by parts on the left, using that $x(T-1)=0$ and $-\lambda^{-1} \alpha \exp (-\lambda)=1$ and multiplying both sides by $\exp [\lambda(T-1 / 2)]$, we get

$$
-\int_{T-1}^{T} x^{\prime}(t) \exp \left[-\lambda\left(t-T+\frac{1}{2}\right)\right] d t=\int_{T}^{\infty}\left[g\left(x_{t}\right)+h\left(x_{t}\right)\right] \exp \left[-\lambda\left(t-T+\frac{1}{2}\right)\right] d t .
$$

The reader may now appreciate our passing from $x\left(t_{n}\right)$ to $x\left(z_{n}+1\right)$ in the arguments above in order to achieve $x(T-1)=0$. Now we consider two cases: $T \leq t_{n}$ and $T>t_{n}$. In the former, we may continue exactly as in [N2, Lemma 2.6] since $x^{\prime}(t) \geq 0$ on $[T-1, T]$ :

$$
\operatorname{Re}\left(\int_{T-1}^{T} x^{\prime}(t) \exp \left[-\lambda\left(t-T+\frac{1}{2}\right)\right] d t\right) \geq \exp \left(-\frac{\mu}{2}\right)\left(\cos \frac{v}{2}\right) x(T) \geq \exp \left(-\frac{\mu}{2}\right)\left(\cos \frac{v}{2}\right) \frac{\delta}{2} .
$$

As the modulus of the right side of (1.6) is less than $\epsilon \delta \mu^{-1} \exp (-\mu / 2)$, we obtain that $\mu / 2 \cos v / 2 \leq \epsilon$, a contradiction to our choice of $\epsilon$.

If $t_{n}<T$, then

$$
\begin{aligned}
& \operatorname{Re}\left(\int_{T-1}^{T} x^{\prime}(t) \exp \left[-\lambda\left(t-T+\frac{1}{2}\right)\right] d t\right) \\
& \quad=\int_{T-1}^{t_{n}} x^{\prime}(t) \exp \left[-\mu\left(t-T+\frac{1}{2}\right)\right] \cos v\left(t-T+\frac{1}{2}\right) d t \\
& \quad+\int_{t_{n}}^{T} x^{\prime}(t) \exp \left[-\mu\left(t-T+\frac{1}{2}\right)\right] \cos v\left(t-T+\frac{1}{2}\right) d t .
\end{aligned}
$$

Note that $x^{\prime}(t) \geq 0$ for $T-1 \leq t \leq t_{n}$ and $x^{\prime}(t) \leq 0$ on $t_{n} \leq t \leq T<z_{n+1}$ so the two integrals have opposite sign. The first integral on the right may be estimated exactly as in the previous case:

$$
\int_{T-1}^{t_{n}} x^{\prime}(t) \exp \left[-\mu\left(t-T+\frac{1}{2}\right)\right] \cos v\left(t-T+\frac{1}{2}\right) d t \geq \exp \left(-\frac{\mu}{2}\right)\left(\cos \frac{v}{2}\right) x\left(t_{n}\right) .
$$


We have for $t_{n} \leq t \leq T$,

$$
\exp \left[-\mu\left(t-T+\frac{1}{2}\right)\right] \cos v\left(t-T+\frac{1}{2}\right) \leq \exp \left[-\mu\left(t_{n}-T+\frac{1}{2}\right)\right] \leq 1
$$

since $T-t_{n}=\left|\tau\left(x\left(t_{n}\right)\right)-\tau(0)\right| \leq q x\left(t_{n}\right) \leq q a<1 / 2$. Hence,

$$
\begin{aligned}
& \int_{t_{n}}^{T} x^{\prime}(t) \exp \left[-\mu\left(t-T+\frac{1}{2}\right)\right] \cos v\left(t-T+\frac{1}{2}\right) d t \geq \int_{t_{n}}^{T} x^{\prime}(t) d t \\
& =x(T)-x\left(t_{n}\right)=x^{\prime}(s)\left(T-t_{n}\right) \geq x^{\prime}(s) q x\left(t_{n}\right) \geq-\operatorname{apqx}\left(t_{n}\right)
\end{aligned}
$$

where $t_{n}<s<T$ and we have used the estimate above for $T-t_{n}$.

Putting (1.7) and (1.8) together we have

$$
\begin{aligned}
\operatorname{Re}\left(\int_{T-1}^{T} x^{\prime}(t) \exp \left[-\lambda\left(t-T+\frac{1}{2}\right)\right] d t\right) & \geq\left(\exp \left(-\frac{\mu}{2}\right)\left(\cos \frac{v}{2}\right)-a p q\right) x\left(t_{n}\right) \\
& \geq \frac{2}{3} \exp \left(-\frac{\mu}{2}\right)\left(\cos \frac{v}{2}\right) x\left(t_{n}\right) \geq \exp \left(-\frac{\mu}{2}\right)\left(\cos \frac{v}{2}\right) \cdot \frac{\delta}{2}
\end{aligned}
$$

A contradiction is obtained exactly as in the previous case.

Theorem 1.8: Assume that (F), (D), (B) and $f^{\prime}(0)>\pi / 2$ hold. Then $T$ has a nonzero fixed point in $K$.

Proof: We show that 0 is an ejective fixed point of $T$ so that our result follows from a Theorem of Browder [Br] (see also [H, Thm. 11.2.4]) on the existence of a nonejective fixed point.

From Lemma $1.7,\left|x\left(t_{n}, \phi\right)\right| \geq a / 2$ for infinitely many integers $n$ if $\phi \in K \backslash 0$. Let $b<a / 2$ be such that $\max \{f(x): 0 \leq x \leq b\}<a /(2 R)$. If $x\left(t_{2 n}, \phi\right)<b$ for every integer $n \geq N$, then

$$
\left|x\left(t_{2 n+1}, \phi\right)\right| \leq \int_{z_{2 n+1}}^{t_{2 n+1}}|f(x(s-\tau(x(s))))| d s<\frac{a}{2 R} \tau\left(x\left(t_{2 n+1}, \phi\right)\right)<a / 2
$$

since $t_{2 n+1}-\tau\left(x\left(t_{2 n+1}\right)\right)=z_{2 n+1}$. This contradiction implies that $x\left(t_{2 n}, \phi\right) \geq b$ for infinitely many $n$. As $\left\|T^{n}(\phi)\right\|=x\left(t_{2 n}, \phi\right)$, we see that 0 is an ejective fixed point of $T$. This proves the theorem. 


\section{Boundedness and Oscillation Properties of Solutions.}

In order to apply our main result, one must find a priori bounds for solutions $x(t, \phi)$ for $\phi$ in a set $\tilde{K}_{N}$. More precisely, we must give sufficient conditions for the existence of $M$ and $N>0$ such that

$$
-M \leq x(t, \phi) \leq N
$$

for all $t$ in the maximal interval of existence of $x(t, \phi)$, for $\phi$ belonging to the set

$$
\tilde{K}_{N}=\left\{\phi \in C_{r}: \phi(-r)=0, \phi \geq 0, \phi \text { nondecreasing, lip } \phi<\infty \text { and } \phi(0) \leq N\right\},
$$

where $r=\max \{\tau(x): 0 \leq x \leq N\}$. As we noted in Lemma 1.2, for $\phi \in \tilde{K}_{N}, t-\tau(x(t, \phi)) \geq$ $-r$ for all $t$ in the interval of existence of $x(\cdot, \phi)$, without assuming (2.1).

We assume throughout this section that (F) and (D) of section one hold.

Define

$$
\begin{aligned}
& F^{+}(u)=\max \{f(x): 0 \leq x \leq u\} \\
& F^{-}(u)=\max \{-f(x):-u \leq x \leq 0\}
\end{aligned}
$$

for $u \geq 0$.

Proposition 2.1: Suppose $M, N>0$ satisfy

$$
\begin{aligned}
& \text { (a) } \frac{M}{\tau(-M)}>F^{+}(N) \\
& \text { (b) } \frac{N}{\tau(N)}>F^{-}(M)
\end{aligned}
$$

Then (2.1) holds for all $\phi \in \tilde{K}_{N}$.

Proof: For $\phi \in \tilde{K}_{N} \backslash 0$, we argue as in Proposition 1.3 (i), (ii) that $x^{\prime}(t, \phi) \leq 0$ for $0 \leq t \leq$ $t_{1} \leq \infty$, where $t_{1}>z_{1}$ and $t_{1}=+\infty$ is not yet ruled out. This argument did not make use of the a priori bound (B). If there exists $t_{0} \in\left(z_{1}, t_{1}\right]$ such that $x(t)>-M$ for $t<t_{0}$ and $x\left(t_{0}\right)=-M$, then $x^{\prime}(s)<0$ so $N \geq x(s-\tau(x(s)))>0$ for $z-1 \leq s<t_{0}$. Hence,

$$
-M=\int_{z_{1}}^{t_{0}} x^{\prime}(s) d s \geq-F^{+}(N)\left(t_{0}-z_{1}\right) .
$$


Since $t_{0}-\tau\left(x\left(t_{0}\right)\right) \leq z_{1}, t_{0}-z \leq \tau(-M)$ so

$$
-M \geq-F^{+}(N) \tau(-M)
$$

contradicting (2.2)(a). Thus, we conclude that $x(t)>-M$ on $\left(z_{1}, z_{2}\right)$. Now suppose that there exists $t_{0} \in\left(z_{1}, t_{2}\right]$ such that $x\left(t_{0}\right)=N$ and $x(t)<N$ for $z_{2} \leq t<t_{0}$. Then

$$
N=\int_{z_{2}}^{t_{0}} x^{\prime}(s) d s \leq F^{-}(M)\left(t_{0}-z_{2}\right) \leq F^{-}(M) \tau(N),
$$

since $-M<x(s-\tau(x(s)))<0$ for $z_{2}<s<t_{0}$ and since $t_{0}-\tau\left(x\left(t_{0}\right)\right)=t_{0}-\tau(N) \leq z_{2}$. Now we have a contradiction to (2.2) (b). Making use of Lemma 1.2, we see this argument can be continued so that (2.1) holds for all $t \geq 0$.

Some special cases where (2.2) hold are worth mentioning here. For example, if there exists $N>0$ such that

$$
\left.\tau( \pm N) \operatorname{lip} f\right|_{[-N, N]}<1,
$$

then (2.2) holds for $M=N$. If $f$ is bounded from below and $\tau$ is bounded from above, then (2.2) holds for all large $N>0$ and $M \geq M(N)>0$, where $M(N)$ depends on $N$. A similar statement holds if $f$ is bounded from above.

In our next result we drop our assumption that $\tau(0)=1$ in order to allow a more appropriate scaling of time. See remark following the proof.

Proposition 2.2: Suppose that $(F)$ and $(D)$ hold for (1.1) and $\tau(x)$ and $f(x)$ satisfy

(i) $\tau_{ \pm}=\lim _{x \rightarrow \pm \infty} \tau(x)$ exist, $0<\tau_{ \pm}<3 / 2, \tau(x)$ is continuously differentiable for large $|x|$ and $\lim _{|x| \rightarrow \infty} x \tau^{\prime}(x)=0 ;$

(ii) $\alpha=\sup \left\{\left|\frac{f(x)}{x}\right|: x \neq 0\right\}<1$.

Then $x(t, \phi)$ is bounded provided that $\phi$ is bounded and lipschitzian on $(-\infty, 0]$. Moreover, there is an $N>0$, such that $|x(t, \phi)| \leq N$ for all $\phi \in \tilde{K}_{N}$.

Proof: Note that $x(t, \phi)$ is defined for all $t \geq 0$ by Lemma 1.1. If the result is false, there is an $x(t)=x(t, \phi)$, such that $\phi$ is bounded and lipschitzian on $(-\infty, 0]$, and $\lim \sup _{t \rightarrow+\infty}|x(t)|=$ 
$\infty$. Denote $\tau=\max \left\{\frac{1}{2}\left(\frac{3}{2}+\tau_{ \pm}\right)\right\}$. Choose $\beta>0, \gamma>0, M>0$, such that for $|x|>M$, $\tau(x)<\tau,\left|x \tau^{\prime}(x)\right|<\beta$, and for $\left|x_{1}\right|>M,\left|x_{2}\right|>M, x_{1} x_{2}>0,\left|\tau\left(x_{1}\right)-\tau\left(x_{2}\right)\right|<\gamma$, where $\beta$ and $\gamma$ are such that $\sqrt{1-4 \tau \beta}-\gamma+\frac{1}{2}>\tau$. Since $\beta$ and $\gamma$ can be chosen arbitrarily small for suitably large $M$, the latter inequality can be assumed.

If $x(t)$ is monotone, then it is easy to see that $x(t)$ must be bounded. Since $x(t)$ is not bounded, it must be oscillatory. Without loss of generality, we assume in the following that there is a $\bar{t}>0$, such that $x(\bar{t})>2 M, x^{\prime}(\bar{t})=0,|x(t)|<x(\bar{t})$ for $t<\bar{t}$. Thus

$$
x(\bar{t}-\tau(x(\bar{t})))=0
$$

Denote

$$
\begin{aligned}
& t_{0}=\bar{t}-\tau(x(\bar{t})) \\
& t_{1}=\max \left\{s, x(s)=\frac{1}{2} x(\bar{t}), x(t)>\frac{1}{2} x(\bar{t}), \text { for } t \in(s, \bar{t}]\right\} .
\end{aligned}
$$

We have

$$
\begin{aligned}
x\left(t_{1}\right)=\frac{1}{2} x(\bar{t}) & =\int_{t_{0}}^{t_{1}}-f(x(t-\tau(x(t)))) d t \\
& <\int_{t_{0}}^{t_{1}} x(\bar{t}) d t=x(\bar{t})\left(t_{1}-t_{0}\right),
\end{aligned}
$$

which indicates that

$$
t_{1}-t_{0}>\frac{1}{2}
$$

Clearly,

$$
\begin{aligned}
\frac{1}{2} x(\bar{t}) & =\int_{t_{1}}^{\bar{t}}-f(x(t-\tau(x(t)))) d t<\int_{t_{1}}^{\bar{t}}|x(t-\tau(x(t)))| d t \\
& =\int_{t_{1}}^{\bar{t}}|x(t-\tau(x(t)))| d(t-\tau(x(t)))+\int_{t_{1}}^{\bar{t}}|x(t-\tau(x(t)))| d \tau(x(t)) .
\end{aligned}
$$

Since $x(\bar{t})>M$, we have $\tau(x(\bar{t}))<\tau$. This leads to $\bar{t}-t_{1}<\tau(x(\bar{t}))<\tau$. Thus

$$
\begin{aligned}
& \int_{t_{1}}^{\bar{t}}|x(t-\tau(x(t)))| d \tau(x(t))=\int_{t_{1}}^{\bar{t}} x^{\prime}(t) \tau^{\prime}(x(t))|x(t-\tau(x(t)))| d t \\
& \quad \leq \int_{t_{1}}^{\bar{t}}\left|x(t) \tau^{\prime}(x(t))\right| \frac{x^{2}(t-\tau(x(t)))}{x(t)} d t \\
& \quad<2 x(\bar{t}) \tau \beta .
\end{aligned}
$$


Since $\left|x^{\prime}(t)\right|=|-f(x(t-\tau(x(t))))|<x(\bar{t})$ for $t \leq \bar{t}$, we have

$$
|x(t)|=\left|x(t)-x\left(t_{0}\right)\right|<x(\bar{t})\left|t-t_{0}\right|, \quad t \leq \bar{t} .
$$

Hence,

$$
\begin{aligned}
& \int_{t_{1}}^{\bar{t}}|x(t-\tau(x(t)))| d(t-\tau(x(t)))=\int_{t_{1}-\tau\left(x\left(t_{1}\right)\right)}^{\bar{t}-\tau(x(\bar{t}))}|x(t)| d t=\int_{t_{1}-\tau\left(x\left(t_{1}\right)\right)}^{t_{0}}|x(t)| d t \\
& <\int_{t_{1}-\tau\left(x\left(t_{1}\right)\right)}^{t_{0}} x(\bar{t})\left(t_{0}-t\right) d t=\frac{1}{2} x(\bar{t})\left(\bar{t}-t_{1}+\tau\left(x\left(t_{1}\right)\right)-\tau(x(\bar{t}))\right)^{2} .
\end{aligned}
$$

Therefore,

$$
\frac{1}{2} x(\bar{t})\left(\bar{t}-t_{1}+\tau\left(x\left(t_{1}\right)-\tau(x(\bar{t}))\right)^{2}+2 x(\bar{t}) \tau \beta>\frac{1}{2} x(\bar{t}),\right.
$$

which implies that

$$
\bar{t}-t_{1}>\sqrt{1-4 \tau \beta}-\gamma
$$

Since $\tau(x(\bar{t}))=\bar{t}-t_{0}=\bar{t}-t_{1}+t_{1}-t_{0}$, we have

$$
\tau(x(\bar{t}))>\sqrt{1-4 \tau \beta}-\gamma+\frac{1}{2}>\tau
$$

a contradiction to the fact that $\tau(x)<\tau$ for $|x|>M$. The conclusion of the second part of the proposition follows by letting $N=2 M$.

By rescaling time appropriately, it is easy to see that if, in Proposition 2.2, we assume the existence of $\tau_{ \pm}>0$ and $0<\alpha<\infty$ and only the single inequality $\alpha \tau_{ \pm}<3 / 2$, then the conclusion of the proposition holds. As a consequence, if $k(1-2)<3 / 2$ in $(0.1)$ with $\tau(x)=\alpha e^{-x^{2}}+(1-\alpha), 0 \leq \alpha<1$, then all solutions are uniformly bounded.

We say that $x(t)$ is oscillatory if $x(t)$ is defined for all large $t$ and has arbitrarily large zeros. It is well known that every solution of

$$
x^{\prime}(t)=-\alpha x(t-\beta)
$$

oscillates if $\alpha>0, \beta>0$ and $\alpha \beta>e^{-1}$. A very nice proof of a more general result is given in [LSS] wherein the following is established. 
Lemma 2.3 [LSS, proof of Theorem]: If $\alpha \beta>e^{-1}$, then there cannot exist a continuously differentiable function $x(t)$ satisfying

$$
x^{\prime}(t)+\alpha x(t-\beta) \leq 0, \quad x(t)>0, \quad x^{\prime}(t)<0
$$

for all large $t$.

Our next result is based on the Lemma.

Theorem 2.4: If $(F)$ and $(D)$ hold and

$$
f^{\prime}(0)>e^{-1},
$$

then every solution of (1.1), defined for $t \geq 0$ and for which $\lim _{t \rightarrow \infty} t-\tau(x(t))=+\infty$, is oscillatory.

Proof: Choose $k$ such that $f^{\prime}(0)>k>e^{-1}$ and $\tau_{0}<1$ with $\tau_{0} k>e^{-1}$. Let $\delta>0$ be such that $|f(x)|>k|x|$ and $\tau(x)>\tau_{0}$ if $|x|<\delta$.

If $x(t)$ is an eventually positive solution, then for large $t, x(t)>0$ and $x^{\prime}(t)<0$. It follows that $\lim _{t \rightarrow \infty} x(t)=\ell \geq 0$ exists and

$$
0=\lim _{t \rightarrow \infty} x^{\prime}(t)=-f\left(\lim _{t \rightarrow \infty} x(t-\tau(x(t)))\right)=-f(\ell)
$$

Thus, $\ell=0$ so that for large $t$ we have $0<x(t)<\delta$ and $x^{\prime}(t)<0$. Then

$$
\begin{aligned}
0 & =x^{\prime}(t)+f(x(t-\tau(x(t))) \\
& >x^{\prime}(t)+k x(t-\tau(x(t))) \\
& >x^{\prime}(t)+k x\left(t-\tau_{0}\right)
\end{aligned}
$$

holds for all large $t$, contradicting Lemma 2.3. 


\section{References}

[A1] W. Alt, Some periodicity criteria for functional differential equations, Manuscripta Math. 23, 1978, 295-318.

[A2] W. Alt, Periodic solutions of some autonomous differential equations with variable time delay, Proc. Conf. on Functional Differential Equations and Approx. of Fixed Points, Bonn 1978. Lecture Notes in Mathematics 730. Springer 1979.

[BM] J. Bélair and M. C. Mackey, Consumer memory and price fluctuations in commodity markets: an integro-differential model, J. Diff. Eqns., 1, 1989, 299-325.

[B] J. Bélair, Population models with state-dependent delays, Proc. 2nd International Conf. on Mathematical Population Dynamics, preprint.

[Br] F. E. Browder, A further generalization of the Schauder fixed point theorem, Duke Math. J., $32,1965,575-578$.

[C] S. N. Chow, Existence of periodic solutions of autonomous functional differential equations, J. Diff. Equations, 15, 1974, 350-378.

[CH] S. N. Chow and J. K. Hale, Periodic solutions of autonomous equations, J. Math. Anal. Appl., 66, 1978, 495-506.

[D] R. D. Driver, Ordinary and Delay Differential Equations, Springer, N.Y.

[G] R. B. Grafton, A periodicity theorem for autonomous functional differential equations, J. Diff. Eqns. 6, 1969, 87-109.

[GY] S. Grossman and J. A. Yorke, Asymptotic behavior and stability criteria for delay differential equations, J. Diff. Eqns. 12, 1972, 236-255.

[HT] K. P. Hadeler and J. Tomiuk, Periodic solutions of difference-differential equations, Arch. Rat. Mech. Anal. 65, 1977, 87-95.

[HY] A. Halanay and J. A. Yorke, Some new results and problems in the theory of differential- 
delay equations, SIAM Rev., 13, 1971, 55-\$0.

[H] J. K. Hale, Theory of Functional Differential Equations, Springer-Verlag, New York, Heidelberg, Berlin, 1977.

[J] G. S. Jones, The existence of periodic solutions of $f^{\prime}(x)=-2 f(x-1)(1+f(x))$, J. Math. Anal. Appl. 5, 1962, 435-450.

[JL] Z. Jackiewicz and E. Lo, The numerical solution of neutral functional differential equations by Adams predictor corrector methods. Tech. report \#118, 1988, Arizona State University.

[KY] J. L. Kaplan and J. A. Yorke, On the nonlinear differential delay equation $x^{\prime}(t)=$ $-f(x(t), x(t-1))$, J. Diff. Eqns. 23, 1977, 293-314.

[LSS] G. Ladas, Y. G. Sficas and I. P. Stavroulakis, Necessary and sufficient conditions for oscillations, Amer. Math. Monthly 90, 1983, 637-640.

[N1] R. D. Nussbaum, Periodic solutions of some nonlinear, autonomous functional differential equations II. J. Diff. Eqns. 14, 1973, 360-394.

[N2] R. D. Nussbaum, Periodic solutions of some nonlinear, autonomous functional differential equations, Ann. Mat. pura appl. IV, Ser. 101, 1974, 263-306.

[W1] H. O. Walther, Existence of a nonconstant periodic solution of a non-linear autonomous functional differential equation representing the growth of a single species population, J. Math. Biol. 1, 1975, 227-240.

[W2] H. O. Walther, On instability, $\omega$-limit sets and periodic solutions of nonlinear autonomous delay equations. In: Functional Differential Equations and Approx. of Fixed Points (H. O. Pertgen and H. O. Walther, eds.) Proc. (Bonn 1978), pp. 489-503. Lecture Notes in Mathematics 730. Springer 1979.

[Wr] E. M. Wright, A nonlinear differential-difference equation, J. Reine Angew. Math. 194, $1955,66-87$.

[MN] J.Mallet-Paret and R. Nussbaum, Periodic solutions of state-dependent differential. delay equations: I. 


\section{Figure Captions}

Figure 1a. Numerical solution of $(0.1)$ with $\alpha=.5, k=2$ and $x(t)=1, t \leq 0$.

Figure 1b. Numerical solution of (0.1) with $\alpha=.5, k=5$ and $x(t)=1, t \leq 0$.

Figure 1c. Numerical solution of (0.1) with $\alpha=.5, k=10$ and $x(t)=1, t \leq 0$.

Figure 2. Numerical solution of (0.3) with $\alpha=2$ and $x(t)=.5, t \leq 0$.

Figure 3. The graph of $x(t, \phi), \phi \in \tilde{K}_{N} \backslash 0$. 


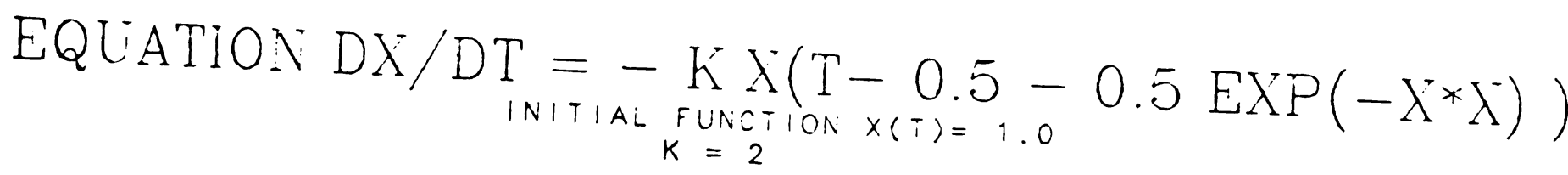

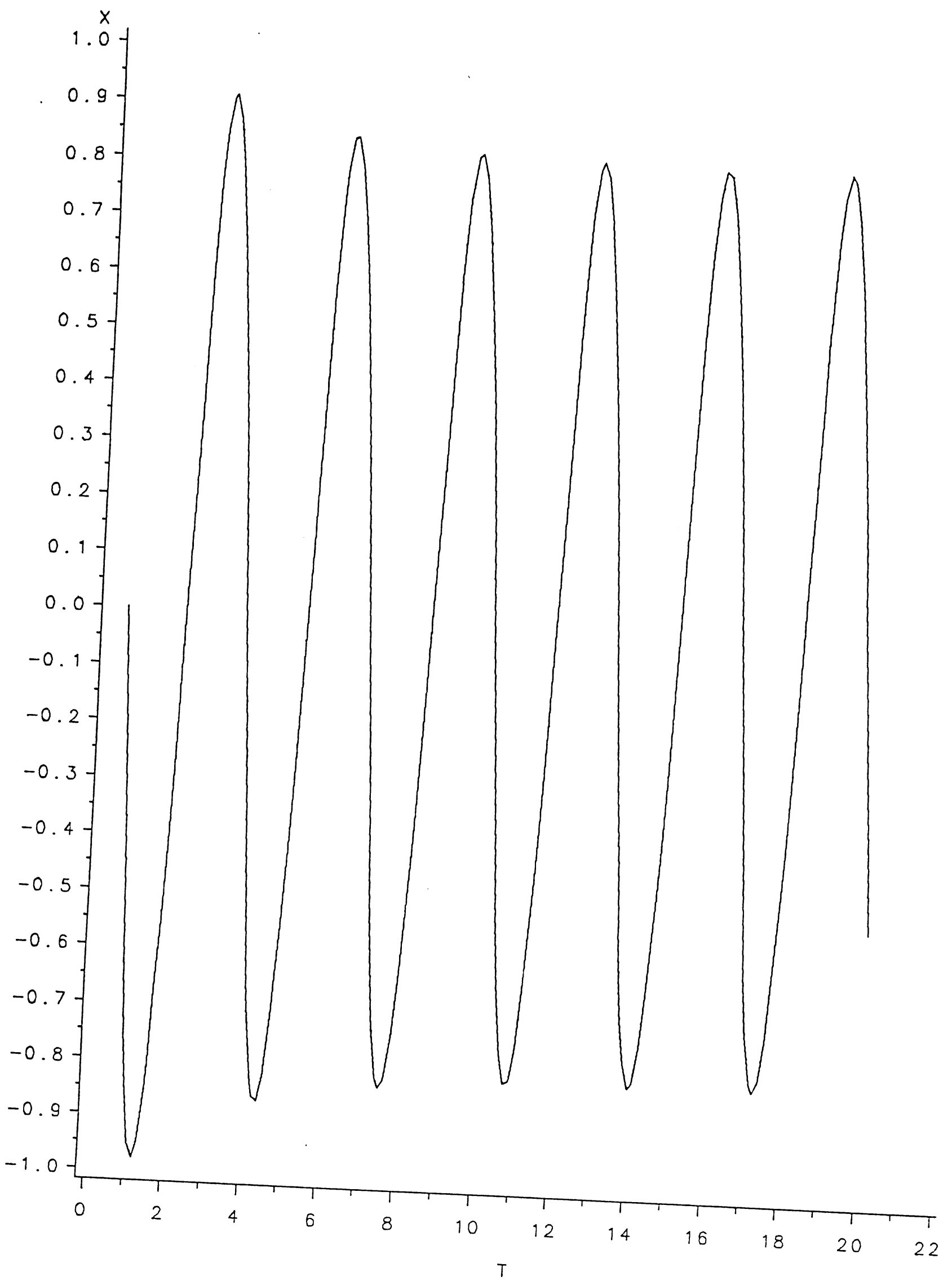




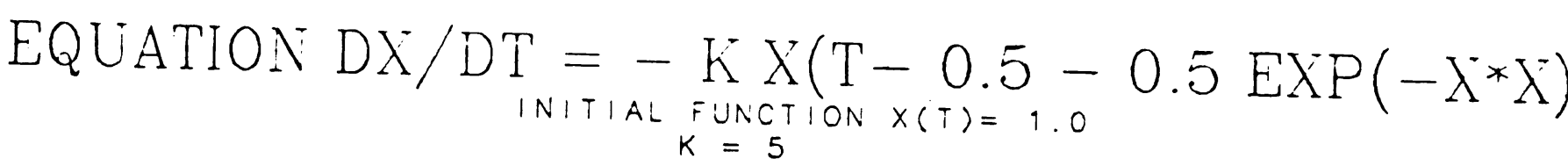

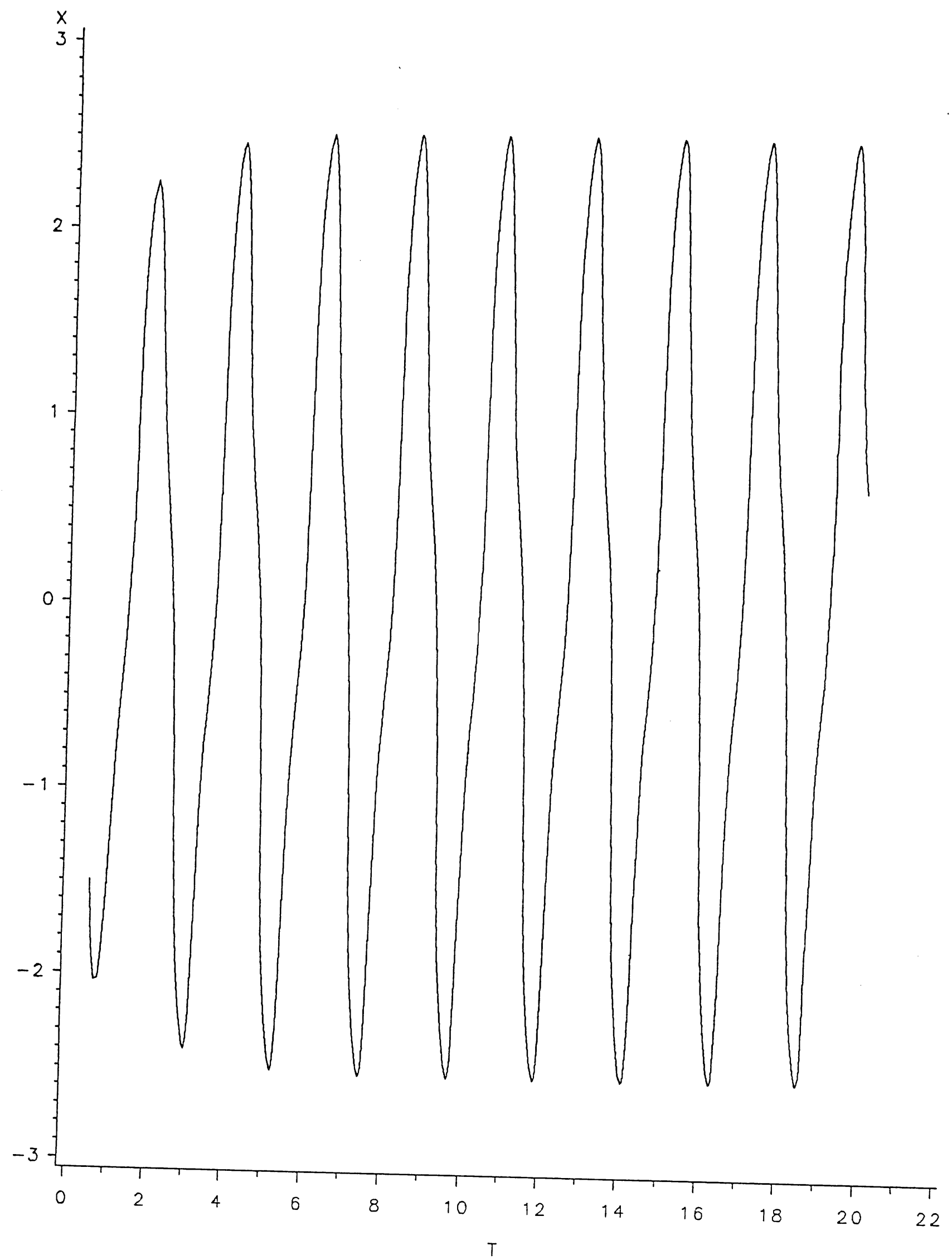


EQUATION DX/DT $=-\underset{\text { INITALL FUNCTION XII)=1.0 }}{\mathrm{K}}\left(\mathrm{T}-0.5 \mathrm{EXP}\left(-\lambda^{*} \mathrm{X}^{*}\right)\right)$ $K=10$

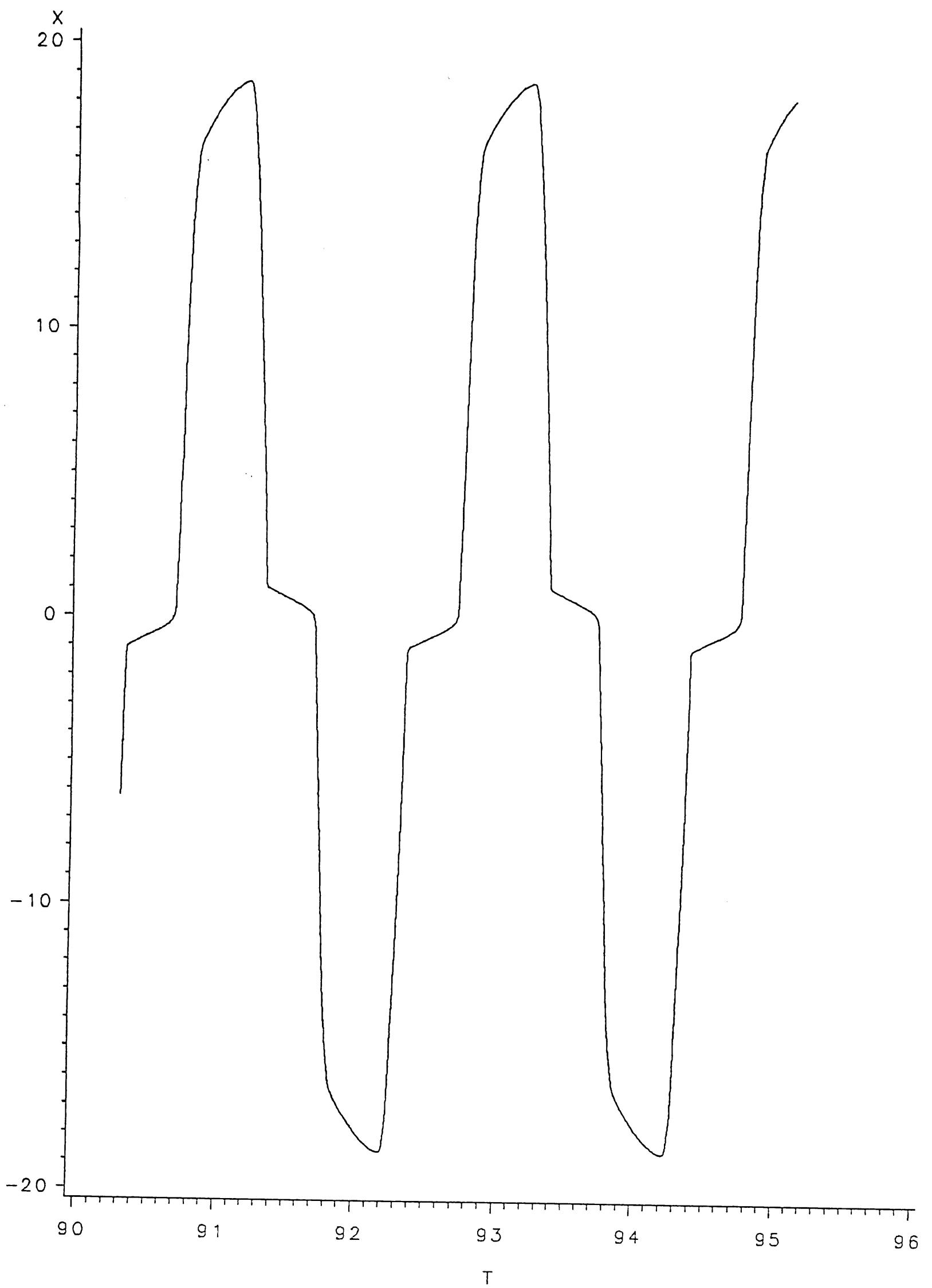




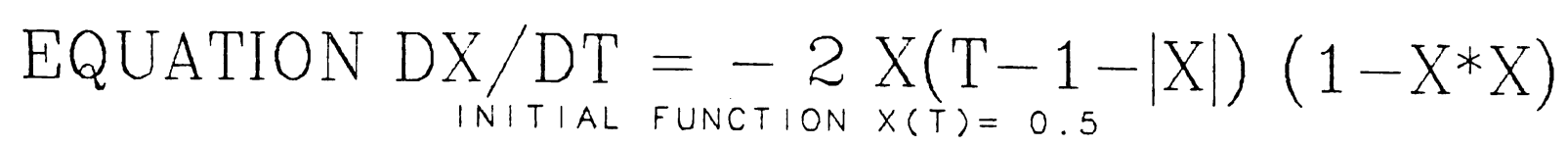

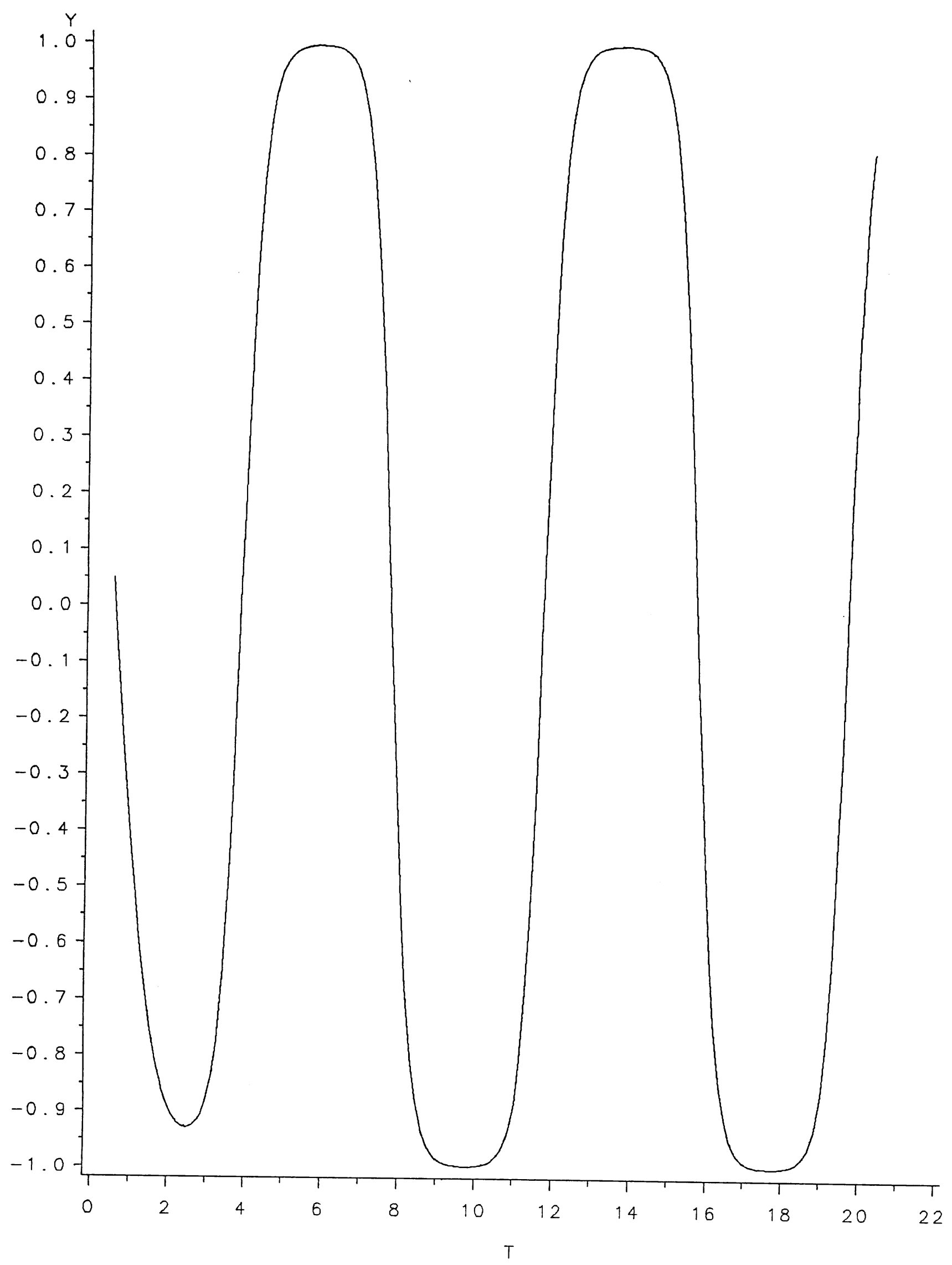




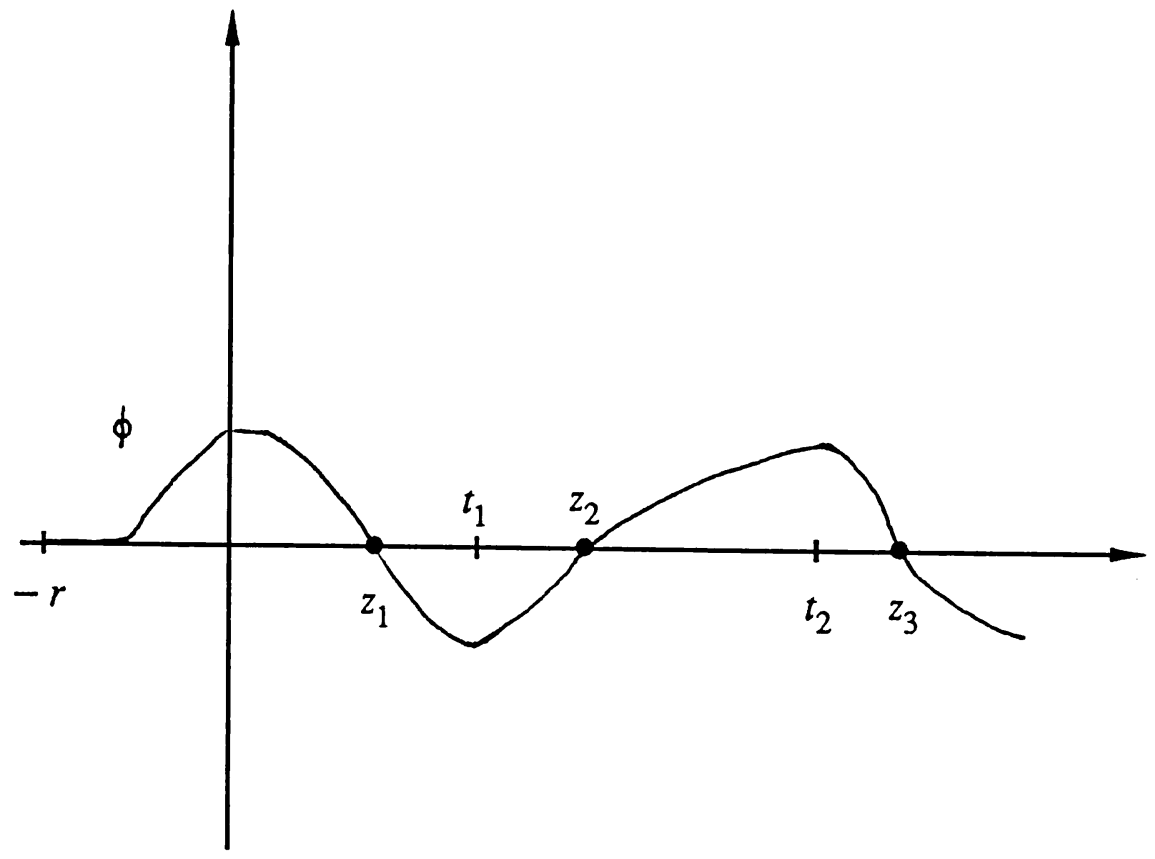

Figure 3: The graph of $x(t, \phi), \phi \in \tilde{K}_{N} \backslash 0$. 